\title{
Escala diagramática para avaliação de severidade da helmintosporiose comum em milho
}

\author{
Diagramatic scale for severity evaluation of common helminthosporium in corn
}

\author{
Adriana Lazaroto ${ }^{\mathrm{I}}$ Idalmir dos Santos ${ }^{\mathrm{II}}$ Valmor Antonio Konflanz ${ }^{\mathrm{III}}$ Gustavo Malagi ${ }^{\mathrm{IV}}$ \\ Rubia Cristiani Camochenav
}

\section{RESUMO}

A helmintosporiose comum, causada pelo fungo Exserohilum turcicum é uma das principais doenças foliares da cultura do milho, na região sul do Brasil, e apresenta um considerável potencial de danos à cultura, especialmente quando o ataque se inicia antes do período de floração. Assim, este trabalho teve como objetivo elaborar e validar uma escala diagramática para avaliação da severidade da helmintosporiose comum do milho. Para isso, foram coletadas folhas com sintomas da doença para a determinação da severidade real, definição dos limites máximos e mínimos, e os níveis intermediários da escala por meio de incrementos logarítmicos, de acordo com a lei de estímulo visual de Weber-Fechner. A escala elaborada sugere sete níveis de severidade: 0,$5 ; 1,0 ; 2,5 ; 6,5 ; 15,5 ; 30,0$; $54,0 \%$. A validação foi realizada por seis avaliadores, sem experiência na quantificação dessa doença, que estimaram a severidade em 30 folhas com diferentes níveis de sintomas, sem e com o auxílio da escala. A utilização da escala diagramática resultou em melhor acurácia e precisão nas estimativas realizadas, sendo uma ferramenta de fácil e rápida utilização, que pode ser adotada para auxiliar na avaliação da severidade da helmintosporiose comum do milho.

Palavras-chave: Zea mays, Exserohilum turcicum, patometria.

\section{ABSTRACT}

The Helminthosporium leaf blight, caused by Exserohilum turcicum, is one of the main spots that attacks corn crop in Southern Brazil. This disease causes considerable economic losses on this crop, especially when the attack begins before the flowering period. The aim of this research was to elaborate and validate a diagrammatic scale to evaluate the severity of the Helminthosporium leaf blight in corn. Corn leaves with symptoms of the disease were collected for measuring the real severity and defining the maximum and minimum limits, as well as the intermediate levels and the logarithmic increments, according to Weber-Fechner's stimulus-response law. The elaborated scale suggested seven severity levels: 0,5 ; 1,$0 ; 2,5 ; 6,5 ; 15,5 ; 30,0$ and $54,0 \%$. The validation was performed by six persons without previous experience to quantify this disease. The severity of 30 leaves with different levels of symptoms was estimated, with and without the help of the scale. The use of the diagrammatic scale resulted in better accuracy and precision in the estimates, being a tool for quick and easy use, which can be adopted to assist in the severity evaluation of the Helminthosporium leaf blight in corn.

Key words: Zea mays, Exserohilum turcicum, pathometry.

\section{INTRODUÇÃO}

Entre as doenças que ocorrem na cultura do milho, a helmintosporiose, causada pelo fungo Exserohilum turcicum (Pass.) K. J. Leonard \& E. G. Suggs, é de ocorrência mundial (CARSON \& VAN

ISecretaria da Agricultura e Abastecimento do estado do Paraná (SEAB), Pato Branco, PR, Brasil.

IIPrograma de Pós-graduação em Agronomia, Laboratório de Fitopatologia, Universidade Tecnológica Federal do Paraná (UTFPR), Via do Conhecimento, Km 01, 85503-390, Pato Branco, PR, Brasil. E-mail: idalmirsantos45@gmail.com. Autor para correspondência.

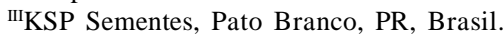

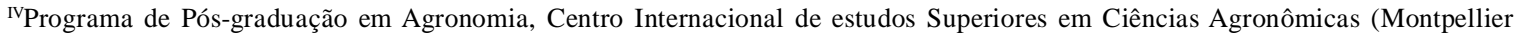

SupAgro), Universidade Federal de Pelotas (UFPel), Pelotas, RS, Brasil.

vPrograma de Pós-graduação em Agronomia, UTFPR, Pato Branco, PR, Brasil. 
DYRE, 1994) e destaca-se por causar mais de $40 \%$ de danos em condições ambientais favoráveis, principalmente, em cultivares suscetíveis. Os sintomas típicos da doença são lesões necróticas, elípticas, medindo de 2,5 a $15 \mathrm{~cm}$ de comprimento, ocorrendo inicialmente nas folhas inferiores, sendo que a coloração do tecido necrosado varia de verde-cinza a marrom (CASELA et al., 2006).

No Brasil, a doença ocorre em maior intensidade em cultivo de milho safrinha, causando os maiores danos quando infecta as plantas no período de floração (FERNANDES \& OLIVEIRA, 2000). No sul do país, a doença é considerada uma das principais manchas foliares incidentes na cultura do milho (REIS et al., 2004).

Nesse contexto, para a quantificação precisa da severidade das doenças foliares, várias estratégias têm sido propostas, sendo uma delas o uso de escalas diagramáticas, as quais tratam de representações ilustradas de uma série de plantas, folhas ou partes de plantas com sinais de doenças em diferentes níveis de severidade (BERGAMIN FILHO \& AMORIM, 1996). Em termos gerais, escalas diagramáticas devem apresentar níveis de severidade reproduzíveis em campo, representar o padrão de evolução das doenças pela estimativa de níveis de dano representativos de todos os estágios de desenvolvimento das doenças e permitir avaliações imediatas (BERGER, 1980).

Para sua elaboração, alguns aspectos devem ser priorizados, tais como: os limites superiores e inferiores da escala, os quais devem corresponder, respectivamente, à quantidade máxima e mínima da doença encontrada no campo; a representação dos sintomas, devendo estar tão próxima quanto possível àqueles observados na planta; e os níveis intermediários da severidade da doença, considerando as limitações de acuidade da visão humana, definidas pela lei de estímulo visual de Weber-Fechner (HORSFALL \& BARRAT, 1945; NUTTER Jr. \& SCHULTZ, 1995), a qual afirma que a percepção da distância entre dois níveis, pela visão humana (acuidade), é aumentada quando a distribuição desses níveis for regulada por incrementos logarítmicos. Além disso, a validação da escala diagramática se faz necessária antes de ser proposta como método padrão de quantificação de doenças, sendo que a obtenção de resultado insatisfatório, durante esse procedimento, deve ser corrigida com a reformulação da escala, ampliando-se, na medida do possível, o número de níveis de severidade.

No Brasil, não existem escalas para avaliação da helmintosporiose comum do milho. As escalas diagramáticas para avaliação da helmintosporiose comum em milho propostas por FULLERTON (1982) e PATAKY (1992) são citadas em diversos trabalhos de pesquisa que avaliam a severidade dessa doença. No entanto, essas escalas foram elaboradas utilizando níveis de severidade que não seguem o acréscimo logarítmico sequencial proposto por HORSFALL \& BARRAT (1945), o que dificulta a distinção visual de cada nível e consequentemente favorece a super ou subestimação da real severidade da doença pelo avaliador. Outro aspecto que limita a utilização segura dessas escalas é a falta de um procedimento de validação, baseado no estudo das variações entre a severidade real e estimada.

Assim sendo, esse trabalho teve como objetivo elaborar e validar uma escala diagramática constituída por níveis de severidade espaçados logaritmicamente, destinada à avaliação da severidade da helmintosporiose comum em milho, causada pelo fungo Exserohilum turcicum.

\section{MATERIAL E MÉTODOS}

A escala diagramática para avaliação de helmintosporiose comum em milho foi elaborada a partir de 125 folhas coletadas em lavouras comerciais na região sudoeste do estado do Paraná, BR, na safra 2009/2010, que apresentavam grande heterogeneidade de incidência da doença. Buscando a padronização do tamanho, cada folha coletada teve seu comprimento total limitado a $20 \mathrm{~cm}$, a fim de facilitar a reprodução das lesões na escala. Além disso, a parte selecionada correspondeu à área da folha em que houve maior manifestação da doença (área representativa da doença).

As áreas lesionadas de cada folha foram copiadas manualmente com tinta preta em lâminas plásticas transparentes e, em seguida, cada folha de milho e a cópia da área lesionada correspondente tiveram suas áreas $\left(\mathrm{cm}^{2}\right)$ determinadas por um medidor de área foliar (marca LICOR, modelo LI3000), sendo a área foliar total (AFT) e a área lesionada total (ALT) de cada folha, utilizadas para o cálculo da severidade real (SR) da doença, pelo

uso da equação $S R(\%)=\frac{A L T}{A F T} * 100$. A partir disso,

foi possível verificar as folhas com menor $(0,4 \%)$ e maior $(54,7 \%)$ severidade, as quais foram utilizadas, respectivamente, para o estabelecimento do limite inferior e superior da escala diagramática.

Para o estabelecimento das severidades intermediárias da escala, quatro tipos de distribuição, respectivamente, com 3, 4, 5 e 6 níveis intermediários, foram simulados a partir de cálculos de incremento logarítmico. Ao fim, a distribuição contendo cinco níveis 
foi selecionada por ter apresentado níveis de severidade melhor espaçados.

Para a construção da escala definida, dentre as 125 folhas coletadas, a folha com menor severidade (limite inferior), a folha com maior severidade (limite superior) e cinco folhas contendo severidades idênticas às severidades simuladas pela distribuição de cinco níveis intermediários foram fotografadas por câmera digital e editadas por um programa de edição de imagens.

A validação da escala proposta foi realizada a partir de 30 folhas de milho com diferentes níveis de severidade da helmintosporiose comum, que foram distribuídas para seis avaliadores sem experiência na avaliação dessa doença. Os avaliadores estimaram visualmente a severidade das lesões em duas etapas: primeiramente, sem a utilização da escala e, em seguida, com a escala. O percentual de área foliar afetada foi determinado pelos avaliadores, considerando como área doente o tecido necrosado.

A acurácia das estimativas visuais de cada avaliador foi avaliada por meio de análise de regressão linear simples, considerando dados com uma distribuição normal, seguindo o modelo $y_{\tau}=b x+a+\varepsilon_{\tau}$, em que $y_{\tau}$ foi considerada a variável dependente (severidade estimada pelos avaliadores); $b$ o coeficiente angular (inclinação da reta); $a$ o coeficiente linear (intercepto); x a variável independente (severidade real da doença); $\varepsilon_{\tau}$ o erro da estimativa com distribuição normal. Considerando esses parâmetros, as hipóteses testadas em cada regressão foram: $H_{0}: a=0 ; b=1 ; H_{1}: a \neq 0 ; b \neq 1$. A precisão das estimativas foi avaliada pelo coeficiente de determinação da regressão $\left(\mathrm{R}^{2}\right)$ e pela variância dos erros absolutos (diferença entre severidade estimada e severidade real).

As análises de regressão linear e dos erros absolutos foram realizadas pelo programa Microsoft Excel 2007. Para verificar a significância dos parâmetros das equações (a e b), realizou-se o teste $t$ $(\mathrm{P} \leq 0,05)$ pelo software computacional $\mathrm{R}(\mathrm{R}$ Development Core Team, 2011).

\section{RESULTADOS E DISCUSSÃO}

As técnicas modernas existentes para a avaliação da severidade das doenças, tais como análise de imagens por ressonância magnética, fotografia infravermelha, termógrafo infravermelho e refletância espectral, são convenientes pela precisão, porém difíceis de serem utilizadas devido à onerososidade (NILSSON, 1995). Dessa maneira, as avaliações visuais de severidade se tornam importantes e o uso de escalas diagramáticas auxilia na diminuição da subjetividade dessas observações visuais (GODOY et al., 1997).

Além disso, a importância de se utilizar uma grande amostragem de folhas para a preparação de uma escala diagramática reflete a possibilidade de inovação na construção dessas escalas, de modo que, nesse trabalho, essa ação contribuiu para que a severidade real de algumas folhas fosse coincidente com as severidades simuladas pelos incrementos logarítmicos, fato este que permitiu a construção da escala proposta pela utilização de fotografias ao invés de desenhos manuais, tradicionalmente utilizados na construção das escalas diagramáticas.

Após um ajuste dos níveis de severidade para intervalos de 0,5 pontos percentuais, a escala proposta neste trabalho apresenta sete níveis de severidade: 0,$5 ; 1,0 ; 2,5 ; 6,5 ; 15,5 ; 30,0 ; 54,0 \%$ (Figura 1). Severidades maiores que $54,0 \%$ proporcionam senescência foliar.

$\mathrm{Na}$ validação da escala, foi possível observar uma considerável melhora nas severidades estimadas com o auxílio da escala, quando comparada às estimativas realizadas sem esse recurso para os seis avaliadores (Figura 2). Os valores estimados para os parâmetros coeficiente linear (a), coeficiente angular (b) e coeficiente de determinação $\left(\mathrm{R}^{2}\right)$, com e sem utilização da escala diagramática estão apresentados na tabela 1.

A acurácia dos avaliadores apresentou melhoria pela utilização da escala. Essa melhoria pode ser verificada pela análise dos coeficientes angulares das regressões obtidos pela estimativa dos avaliadores, que não diferiram significativamente de 1 após a utilização da escala, indicando que as tendências estimadas de severidade passaram a ser significativamente iguais às tendências de severidade real da doença a partir da utilização da escala, de acordo com VALE et al. (2004). Nesta análise, apenas as tendências formadas a partir dos dados de avaliação do avaliador B apresentaram coeficientes angulares significativamente iguais às severidades reais, antes e depois da utilização da escala, sendo este resultado possível, em razão de um menor estímulo do incremento logarítmico sobre a visão deste avaliador. As médias dos coeficientes angulares das retas foram 3,14 e 0,95, antes e depois da utilização da escala, respectivamente. Os valores dos coeficientes lineares, não apresentaram diferenças significativas de zero, antes e depois da utilização da escala e, neste caso, não auxiliaram na verificação da acurácia dos avaliadores.

Analisando a precisão dos avaliadores pelo coeficiente de determinação $\left(\mathrm{R}^{2}\right)$ das equações de regressão, observaram-se valores entre 0,63 e 0,85 , com 


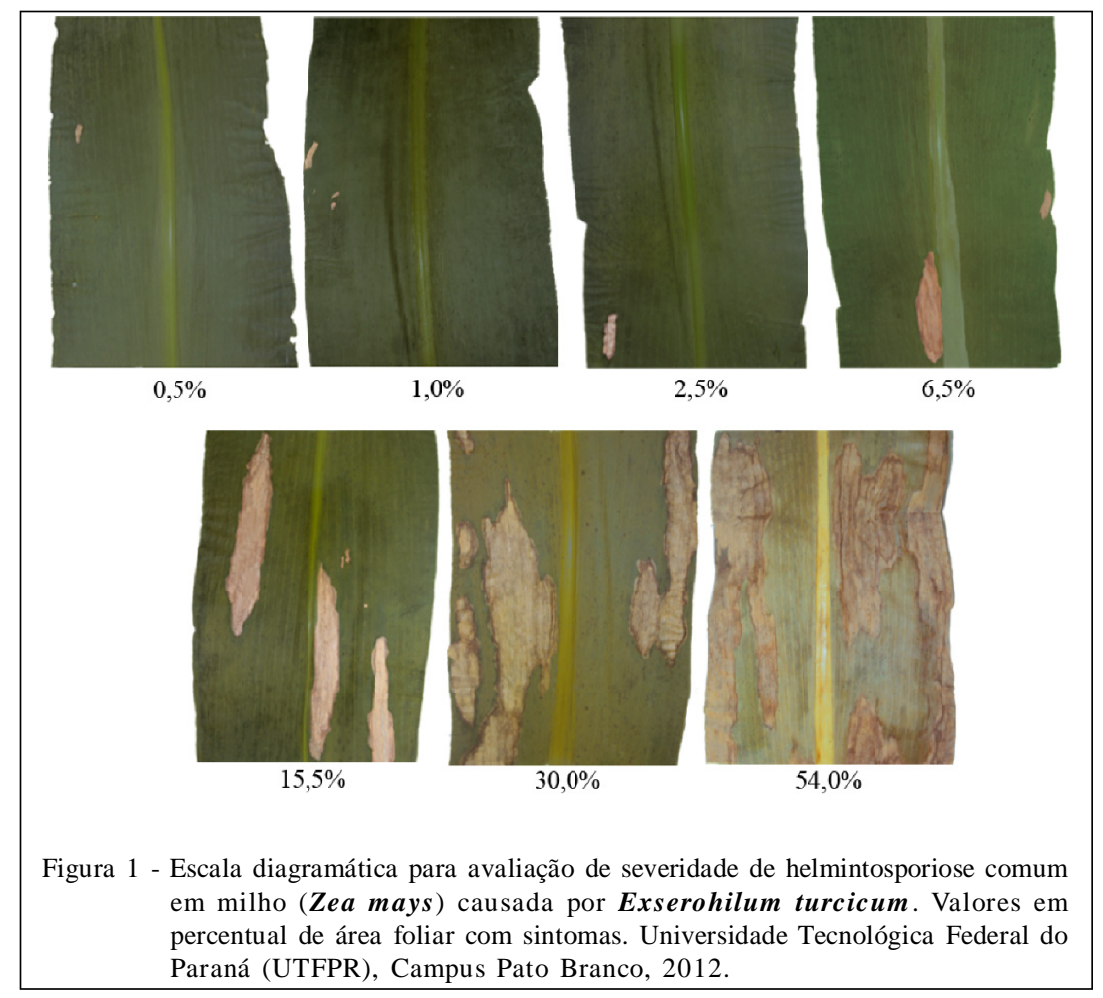

média de 0,76 , para a estimativa sem escala, e valores entre 0,86 e 0,96 , com média de 0,90 , para a estimativa com o uso da escala. Conforme descrito por (NUTTER Jr \& SCHULTZ, 1995), quanto mais próximo de 1 (ou $100 \%$ ) se apresenta o coeficiente de determinação da regressão, mais precisa é a estimativa. Assim, o aumento expressivo nos valores de $\mathrm{R}^{2}$ observados pelo uso da escala diagramática para avaliação da helmintosporiose indica maior precisão dos avaliadores e maior confiabilidade na quantificação dessa doença. Mesmo com o uso da escala, houve uma tendência de sub ou superestimação da doença, mas estudos mostram que cada indivíduo possui uma percepção visual diferente para avaliação das doenças (SPÓSITO et al., 2004).

Os erros absolutos dos avaliadores, sem e com a escala diagramática, podem ser observados na figura 2. Nas avaliações sem auxílio da escala diagramática proposta, o erro absoluto dos avaliadores apresentou dispersão entre $-13,72 \%$ e $+18,95 \%$, enquanto que a variação pelo uso da escala passou a ser entre $-10,13 \%$ e $+10,22 \%$. Segundo os critérios adotados por programas de computadores para treinamento na quantificação de doenças, como, por exemplo, o Distrain (TOMERLIN \& HOWELL, 1988) e Disease Pro (NUTTER Jr \& WORAWITLIKIT, 1989), a variação na estimação da severidade entre $-10 \%$ e $+10 \%$ é considerada aceitável. Fazendo a comparação das avaliações sem e com uso da escala diagramática proposta para quantificação de helmintosporiose comum em milho, observou-se que, de modo geral, o erro diminuiu para todos os avaliadores.

Na escala para avaliação da helmintosporiose comum do milho, elaborada por Fullerton, em 1982, foi utilizada somente análise de regressão para avaliar os dados, sendo que ela não foi validada com a utilização de avaliadores sem experiência, o que poderia dar mais credibilidade e maior precisão nas estimativas de danos e perdas na lavoura. Qualquer procedimento de quantificação de doenças deve ser acurado e preciso para que a melhor estratégia de controle possa ser adotada.

No entanto, as escalas utilizadas atualmente para avaliar a helmintosporiose do milho podem induzir a erros de magnitude desconhecidos e impedir uma padronização correta da avaliação. Em vários trabalhos encontrados na literatura, como o de VIEIRA et al. (2009), que avaliou o desempenho agronômico de milho pipoca, e PATAKY (1992), o qual estudou a relação da helmintosporiose com o rendimento e o teor de açúcar presente no milho, foram utilizadas escalas adaptadas ou modificadas, ou software de treinamento. A maior parte dessas escalas não foram elaboradas considerando a lei do estímulo de Weber-Fechner. No trabalho de FULLERTON (1982), uma escala foi 

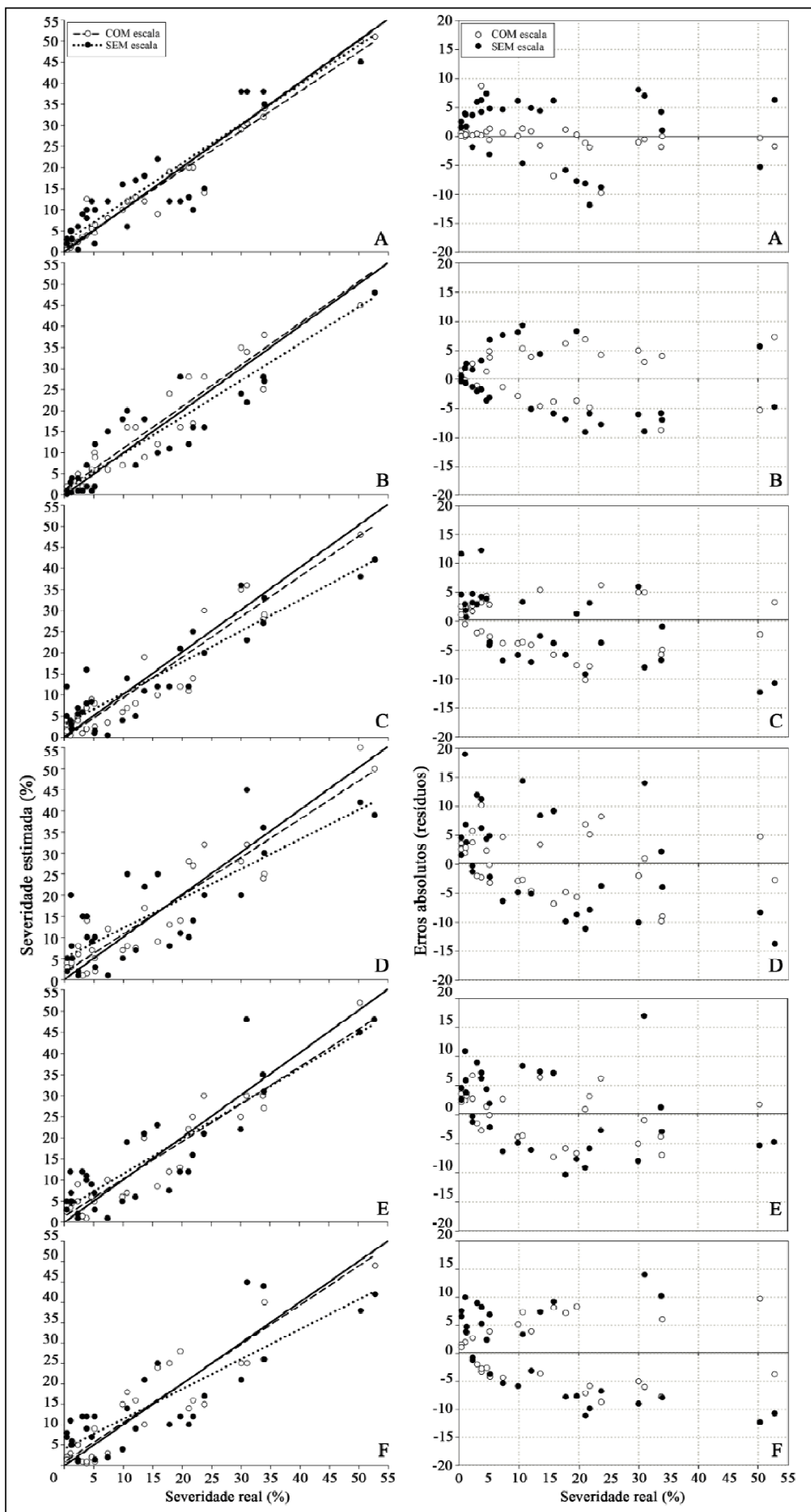

Figura 2 - Severidade estimada (à esquerda) e erros absolutos (severidade real severidade estimada) (à direita) obtidos por seis avaliadores sem experiência em avaliação de doenças, com e sem o uso da escala proposta: avaliador 1(A), 2(B), 3(C), 4(D), 5(E) e 6(F). Nas estimativas de severidade, a linha contínua representa a situação ideal, em que as estimativas são idênticas à real; a linha pontilhada grossa ( - - - ) representa a severidade estimada com o uso da escala e a linha pontilhada fina ( $\cdot * *$ ) a severidade estimada sem o uso da escala. Para os erros absolutos, os pontos com preenchimento ( $\bullet$ ) e sem preenchimento ( $\bullet$ ) foram obtidos com e sem o uso da escala, respectivamente. Universidade Tecnológica Federal do Paraná (UTFPR), Campus Pato Branco, 2012. 
Tabela 1 - Coeficientes lineares (a), coeficientes angulares (b) e coeficientes de determinação $\left(R^{2}\right)$ de equações de regressão linear simples e suas médias, relacionando estimativas visuais de severidade efetuadas por seis avaliadores (A, B, C, D, E e F), sem e com o uso da escala diagramática proposta, com a severidade real a partir de 30 folhas de milho atacadas por helmintosperiose comum do milho. Universidade Tecnológica Federal do Paraná (UTFPR), Campus Pato Branco, 2012.

\begin{tabular}{|c|c|c|c|c|c|c|}
\hline \multirow{2}{*}{ Avaliador } & \multicolumn{6}{|c|}{---Sem escala---- } \\
\hline & a & $\mathrm{b}$ & $\mathrm{R}^{2}$ & $\mathrm{a}$ & $\mathrm{b}$ & $\mathrm{R}^{2}$ \\
\hline A & $0,93 * *$ & $2,39 *$ & 0,85 & $0,93 * *$ & $0,65^{\mathrm{ns}}$ & 0,96 \\
\hline B & $0,87 * *$ & $1,04^{\mathrm{ns}}$ & 0,84 & $0,98 * *$ & $1,22^{\mathrm{ns}}$ & 0,92 \\
\hline $\mathrm{C}$ & $0,73 * *$ & $2,99 *$ & 0,82 & $0,95 * *$ & $-0,20^{\mathrm{ns}}$ & 0,90 \\
\hline $\mathrm{D}$ & $0,70 * *$ & $5,23 * *$ & 0,63 & $0,90 * *$ & $1,78^{\mathrm{ns}}$ & 0,87 \\
\hline E & $0,83 * *$ & $3,12 *$ & 0,77 & $0,88 * *$ & $1,39^{\mathrm{ns}}$ & 0,90 \\
\hline $\mathrm{F}$ & $0,73 * *$ & $4,07 * *$ & 0,69 & $0,96 * *$ & $0,90^{\mathrm{ns}}$ & 0,86 \\
\hline Média & 0,79 & 3,14 & 0,76 & 0,93 & 0,95 & 0,90 \\
\hline
\end{tabular}

* e ** - situações em que a hipótese nula $(\mathrm{a}=0 \mathrm{ou} \mathrm{b}=1)$ foi rejeitada pelo teste $t$, respectivamente, ao nível de significância de 1 e $5 \%$ de erro tipo I. ${ }^{\text {ns }}$ - diferença não significativa.

desenvolvida calculando-se a porcentagem de área foliar afetada pela helmitosporiose, obtendo-se uma média por planta, com valores convertidos para percentual de área foliar afetado pela doença em uma população. Todavia, as notas sugeridas representam percentuais crescentes de dano da doença, sobre a área foliar de toda planta, sem utilização de incrementos logarítmicos, o que dificulta a sua utilização. A escala de Cobb, elaborada para quantificação da ferrugem da folha em trigo (Puccinia triticina Erikss) é também utilizada para quantificação de doenças foliares do milho, porém o seu uso não garante precisão experimental na quantificação da helmintosporiose (PINHO et al., 2000).

Portanto, verifica-se que a avaliação da helmintosporiose do milho tem sido realizada com a utilização de diversas escalas com adaptações e inadequações, dificultando a precisão e a padronização das avaliações da referida doença. Nesse sentido, a escala proposta neste trabalho padroniza o procedimento de quantificação da helmintosporiose comum em milho, contribui para a precisão das avaliações e com a qualidade dos trabalhos realizados, envolvendo a quantificação dessa doença.

\section{CONCLUSÃO}

Pelo trabalho desenvolvido, foi possível estabelecer uma escala diagramática para avaliação da helmintosporiose comum em milho, sendo que a quantificação acurada e precisa dos sintomas pelo uso dessa escala, permitem validá-la e recomendá-la como ferramenta nos estudos envolvendo esta doença.

\section{REFERÊNCIAS}

BERGAMIN FILHO, A.; AMORIM, L. Doenças de plantas tropicais: epidemiologia e controle econômico. Piracicaba. Agronômica Ceres, 1996. 299p.

BERGER, R.D. Measuring disease intensity. In: TENG, P.S.; KRUPA, S.V. (Eds.). Crop loss assessment. St. Paul. University of Minnesota, 1980. p.28-31.

CARSON, M.L.; VAN DYRE, C.G. Effect of light and temperature on expression of partial resistance of maize to Exserohilum turcicum. Plant Disease, v.78, n.5, p.519-522, 1994.

CASELA, C.R. et al. Doenças na cultura do milho. Sete Lagoas, MG: Embrapa Milho e Sorgo, 2006. 14p. (Circular Técnica, 83).

FERNANDES, F.T.; OLIVEIRA, E. Principais doenças na cultura do milho. Sete Lagoas: EMBRAPA/CNPMS, 2000. 80p.

FULLERTON, R.A. Assessment of leaf damage caused by northern leaf blight in maize. New Zealand Journal of Experimental Agriculture, v.10, n.3, p.313-316, 1982. Disponível em: <http://www.tandfonline.com/doi/abs/10.1080/ 03015521.1982.10427890>. Acesso em: 14 maio, 2012. doi: 10.1080/03015521.1982.10427890.

GODOY, C.V. et al. Diagramatic scales for bean diseases: development and validation. Zeitschrift fur Pflanzenkrankheiten und Pflanzenschutz, v.104, n.4, p.336-345, 1997.

HORSFALL, J.C.; BARRATT, R.W. An improved grading system for measuring plant diseases. Phytopathology, v.35, p.665, 1945.

NILSSON, H.E. Remote sensing and image analysis in plant pathology. Canadian Journal of Plant Pathology, v.17, p.154-166, 1995.

Ciência Rural, v.42, n.12, dez, 2012. 
NUTTER JÚNIOR, F.W.; SCHULTZ, P.M. Improving the accuracy and precision of disease assessments: selection of methods and use of computer-aided training programs. Canadian Journal of Plant Pathology. v.17, p.174-184, 1995.

NUTTER JÚNIOR, F.W.; WORAWITLIKIT, O. Disease.Pro: a computer program for evaluating and improving a person ability to assess disease proportion. Phytopathology, v.79, p. $1135,1989$.

PATAKY, J.K. Relationships between yield of sweet corn and northern leaf blight caused by Exserohilum turcicum. Phytopathology, v.82, p.370-375, 1992.

PINHO, R.G.V. et al. Comparação de métodos para a quantificação da severidade das ferrugens polissora e tropical do milho. Ciência e Agrotecnologia, v.24, n.1, p.22-37, 2000.

R DEVELOPMENT CORE TEAM. R: a language and environment for statistical computing. Vienna, Austria, 2011. Disponível em: <http://www.r-project.org>. Acesso em: 05 julho, 2010.
REIS, E.M.; CASA, R.T. Manual de identificação e controle de doenças de milho. Lavras: Ciências Agrotécnicas, 2004. 331p.

SPÓSITO, M.B. et al. Elaboração e validação de escala diagramática para avaliação da severidade da mancha preta em frutos cítricos. Fitopatologia Brasileira, v.29, p.81-85, 2004. Disponível em: <http://www.scielo.br/pdf/fb/v29n1/a12v29n1.pdf>. Acesso em: 14 maio, 2012. doi: 10.1590/S0100-41582004000100012.

TOMERLIN, J.; HOWELL, T.A. Distrain: a computer program for training people to estimate disease severity on cereal leaves. Pilam Disease, v.72, p.455-459, 1988.

VALE, F.X.R. et al. Quantificação de doenças e do crescimento do hospedeiro. In: VALE, F.X.R. et al. Epidemiologia aplicada ao manejo de doenças de plantas. Belo Horizonte: Perfil, 2004. v.1, cap.3, p.91-126.

VIEIRA, R.A. et al. Desempenho agronômico de novos híbridos de milho-pipoca no noroeste do estado do Paraná, Brasil. Acta Scientiarum Agronomy, v.31, n.1, p.29-36, 2009. 